\title{
Activity Propagation in an Avian Basal Ganglia- Thalamocortical Circuit Essential for Vocal Learning
}

\author{
Satoshi Kojima and Allison J. Doupe \\ Departments of Physiology and Psychiatry, Keck Center for Integrative Neuroscience, University of California, San Francisco, San Francisco, California \\ 94143-0444
}

In mammalian basal ganglia-thalamocortical circuits, GABAergic pallidal neurons are thought to "gate" or modulate excitation in thalamus with their strong inhibitory inputs and thus signal to cortex by pausing and permitting thalamic neurons to fire in response to excitatory drive. In contrast, in a homologous circuit specialized for vocal learning in songbirds, evidence suggests that pallidal neurons signal by eliciting postinhibitory rebound spikes in thalamus, which could occur even without any excitatory drive to thalamic neurons. To test whether songbird pallidal neurons can also communicate with thalamus by gating excitatory drive, as well as by postinhibitory rebound, we examined the activity of thalamic relay neurons in response to acute inactivation of the basal ganglia structure Area X; Area X contains the pallidal neurons that project to thalamus. Although inactivation of Area X should eliminate rebound-mediated spiking in thalamus, this manipulation tonically increased the firing rate of thalamic relay neurons, providing evidence that songbird pallidal neurons can gate tonic thalamic excitatory drive. We also found that the increased thalamic activity was fed forward to its target in the avian equivalent of cortex, which includes neurons that project to the vocal premotor area. These data raise the possibility that basal ganglia circuits can signal to cortex through thalamus both by generating postinhibitory rebound and by gating excitatory drive and may switch between these modes depending on the statistics of pallidal firing. Moreover, these findings provide insight into the strikingly different disruptive effects of basal ganglia and cortical lesions on songbird vocal learning.

\section{Introduction}

The anterior forebrain pathway (AFP) of songbirds is recognized as a basal ganglia-thalamocortical circuit, based on anatomy, physiology, and embryology (see Fig. 1A) (Bottjer and Johnson, 1997; Reiner et al., 2004b; Person et al., 2008). Unlike its mammalian counterparts and the rest of songbird basal ganglia, which subserve a broad range of motor behaviors, the AFP is a discrete circuit specialized for the well defined sensorimotor tasks of vocal learning and production. The AFP thus provides a simple model for information processing in basal ganglia circuits.

Recently, however, examination of the GABAergic inhibitory pallidothalamic connection of birds, from the striatopallidal nucleus Area X to the medial portion of the dorsolateral nucleus of the anterior thalamus (DLM), has suggested a new model of activity propagation through the basal ganglia circuit, which is different from the classical model originally proposed in mammals (MacLeod et al., 1980; Deniau and Chevalier, 1985; Inase et al.,

Received 0ct. 8, 2008; revised March 6, 2009; accepted March 12, 2009

This work was supported by National Institutes of Health Grants MH055987 and MH550012 (to A.J.D.). We thank Loren Frank and Brian Wright for their helpful suggestions regarding analysis methods, Michael Brainard and Mimi Kao for their thoughtful comments on this manuscript, and Adria Arteseros for expert histological assistance.

Correspondence should be addressed to Satoshi Kojima, Departments of Physiology and Psychiatry, Keck Center for Integrative Neuroscience, University of California, San Francisco, San Francisco, CA 94143-0444. E-mail: skojima@phy.ucsf.edu.

D01:10.1523/JNEUROSCI.4903-08.2009

Copyright $\odot 2009$ Society for Neuroscience $\quad$ 0270-6474/09/294782-12\$15.00/0
1996). By analogy to the classical model, Area X pallidal neurons were initially assumed to gate excitation in DLM (thalamic) target neurons so that pauses in pallidal firing would enable the excitatory drive to generate DLM firing ("gating model") (Luo and Perkel, 1999a,b; Mooney et al., 2002). However, Person and Perkel (2005) showed that avian pallidothalamic signaling is more complex: they found in vitro that DLM neurons were quiescent but could be directly driven to spike by postinhibitory rebound, evoked after a rapid deceleration of high-frequency pallidal input. They then demonstrated in vivo that DLM spikes follow similar decelerations in the high-frequency firing of Area $\mathrm{X}$ pallidal neurons (Person and Perkel, 2007). These results suggest the new model that Area X pallidal neurons signal to thalamus primarily by generating postinhibitory rebound in DLM neurons ("postinhibitory rebound model") (see Fig. $1 B_{1}$, intact circuit) and raised the possibility of a similar signaling mechanism in the mammalian pallidothalamic connection as well.

Although the postinhibitory rebound model could account for much of DLM firing (Person and Perkel, 2007), it does not by itself disprove the gating model: DLM neurons in vivo may have intrinsic or extrinsic excitatory drive not seen in vitro and thus generate spikes driven by such excitatory drive (possibly after generating rebound spikes) when pallidal neurons have long pauses in their firing or are inactivated, as in mammals (see Fig. $1 B_{2}$, right panel) (MacLeod et al., 1980; Deniau and Chevalier, 1985; Inase et al., 1996). Evidence for such gating in birds would 
strengthen the parallels between avian and mammalian basal ganglia circuits and would also suggest that pallidal neurons can signal to thalamus using two distinct mechanisms, i.e., by gating excitatory drive to thalamus and by generating postinhibitory rebound. Conversely, avian pallidal neurons, at least in anesthetized animals, could drive thalamic neurons exclusively by postinhibitory rebound and not by gating excitatory drive, which would be strikingly different from mammalian basal ganglia circuits. In this case, Area X inactivation should lead to decreased thalamic activity (see Fig. $1 B_{2}$, left panel), because of loss of the temporal modulation of pallidal firing required to reset the rebound mechanism.

We therefore investigated pallidothalamic signaling of songbirds by pharmacologically inactivating Area X of adult zebra finches and examining how DLM neurons changed their spontaneous activity. These experiments also allowed us to test how changes in pallidal firing are fed forward to their target in the avian equivalent of cortex, pallium, specifically the lateral magnocellular nucleus of the anterior nidopallium, LMAN (Reiner et al., 2004a) (most so-called pallial areas in birds have an embryological origin similar to mammalian cortex and although not layered, share numerous functional similarities with cortex). The question of how pallidal firing influences cortical activity is still little examined in mammals (but see Paz et al., 2007) but is critical to understanding how basal ganglia circuitry ultimately influences behavioral output. It is especially topical in the AFP, because lesions of Area X and of its cortical target LMAN differ dramatically in their effects on song learning (Bottjer et al., 1984; Sohrabji et al., 1990; Scharff and Nottebohm, 1991).

\section{Materials and Methods}

Experiments were performed on adult (>100-d-old) male zebra finches (Taeniopygia guttata), which were bred in our colony. The care and treatment of experimental animals was reviewed and approved by the animal care and use committee at the University of California, San Francisco (UCSF).

Electrophysiology. Simultaneous extracellular recordings of spontaneous activity from Area X and either DLM or LMAN neurons were performed. Two to fourteen days before the experiment, birds were prepared for neurophysiological recordings by affixing a head post to the skull under Equithesin anesthesia (Solis and Doupe, 1999) and marking the location of the song nuclei on the skull (for details, see Solis and Doupe, 1999). On the day of the experiment, they were anesthetized with a $20 \%$ solution of urethane $(60-85 \mu \mathrm{l}$, i.m.; Sigma), secured in the stereotaxic apparatus by the head post, and placed in a double-walled anechoic sound-attenuating chamber. Body temperature was maintained at $\sim 40^{\circ} \mathrm{C}$ with a temperature controller. Extracellular activity recorded with tungsten electrodes $(2-8 \mathrm{M} \Omega$ ) was amplified and filtered between $300 \mathrm{~Hz}$ and $5 \mathrm{kHz}$ (A-M Systems). Spikes were sorted offline using spikesorting software developed by Michael Lewicki (California Institute of Technology) and Brian Wright (UCSF) (Kojima and Doupe, 2007). Briefly, spike models were constructed from recorded spike waveforms, and spikes were classified using the spike models with a templatematching algorithm based on Bayesian probability theory (Lewicki, 1994). All neurons were judged to be single units based on inspection of the waveforms and by analysis of interspike interval (ISI) violations; any sites where occurrence of ISIs $<0.7 \mathrm{~ms}$ constituted $>1.0 \%$ of total ISI occurrences were discarded.

At some sites in DLM and LMAN, we simultaneously recorded two units on a single electrode, and in DLM, these were paired recordings of a single pallidal axon terminal and a single DLM projection neuron, as previously reported by Person and Perkel (2007). In these recordings, the larger units with their very low-frequency spontaneous firing rates and biphasic action potential spike waveforms were identified as DLM projection neurons, and the smaller units with distinctively higher spontaneous firing rates and relatively asymmetric (monophasic-like) spike waveforms were assumed to be axon terminals of Area $\mathrm{X}$ pallidal neurons (see Fig. 2A), following Person and Perkel (2007).

At the end of each experiment, electrolytic lesions were made at selected locations for reconstructing recording sites. The bird was then deeply anesthetized with Metofane (Pitman-Moore) or Isoflurane (Abott Laboratories) and transcardially perfused with $0.9 \%$ saline, followed by $3.7 \%$ formaldehyde in $0.025 \mathrm{~m}$ phosphate buffer. Brains were postfixed, and $40 \mu \mathrm{m}$ sections were cut with a freezing microtome. Sections were stained with cresyl violet, and electrode tracks and lesions were identified. Only neurons histologically confirmed to be in DLM or LMAN were analyzed.

Antidromic identification of LMAN-projecting DLM neurons and robust nucleus of the arcopallium-projecting LMAN neurons. Antidromic identification of projection neurons in DLM and LMAN was performed by electrical stimulation of their target nuclei LMAN and the robust nucleus of the arcopallium (RA), respectively (Hahnloser et al., 2002; Hahnloser and Fee, 2007). Bipolar stimulating electrodes spaced $0.5 \mathrm{~mm}$ apart were placed in the target nucleus, and electrical stimulation $(0.2 \mathrm{~ms}$ duration, $50-200 \mu \mathrm{A}$ monophasic pulses) was applied either at $0.5-1 \mathrm{~Hz}$ or sporadically. Evoked spike responses were recorded from DLM or LMAN with tungsten microelectrodes. Once a cell was isolated, latencies from stimulus onset to the first antidromically evoked spike were measured for at least 10 stimulus trials, and neurons that had short latency to spiking in response to stimulation, and very low variability in this latency to spike (SD of latency $<100 \mu \mathrm{s}$ ), were judged to be projecting neurons. For most neurons, antidromic activation was then confirmed by a (more stringent) collision test in which the target nucleus was stimulated with some delay after a spontaneous spike in the recorded neuron: consistent with the identification of activated neurons as putative projection neurons, stimulating the target nucleus at a small delay $(0.5-2 \mathrm{~ms})$ after spontaneous spikes resulted in 100\% failure of the antidromic spike (collisions).

LMAN neurons that were not antidromically identified are also likely to be projection neurons because of the following: their mean firing rates in baseline or GABA-response trials were not significantly different from those in the identified LMAN neurons (baseline trials: mean \pm SEM, $3.16 \pm 4.43 \mathrm{~Hz}$ in unidentified neurons, and $1.01 \pm 0.23 \mathrm{~Hz}$ in identified neurons; GABA-response trials: $8.67 \pm 21.16 \mathrm{~Hz}$ in unidentified neurons, and $7.82 \pm 12.46 \mathrm{~Hz}$ in identified neurons; unpaired $t$ test, $p>$ $0.05)$; LMAN neurons recorded in previous anesthetized and chronic extracellular recordings, including a subset identified antidromically as projection neurons (Olveczky et al., 2005), appear to be a homogeneous population of neurons with firing properties similar to the ones here; and similar neurons identified intracellularly, both in vitro and in vivo, also proved to be projection neurons, not interneurons (Livingston and Mooney, 1997; Boettiger and Doupe, 1998; Rosen and Mooney, 2000).

GABA infusion. During simultaneous recordings from Area $\mathrm{X}$ and DLM or Area X and LMAN, a 250 or 500 mM GABA (Sigma) solution was pressure-injected into Area X with a Pressure Micro-Injector (Dagan Corporation) through a graduated glass pipette (10-20 $\mu \mathrm{m}$ tip). Typically, a small volume $(0.1-1.0 \mu \mathrm{l})$ was slowly infused over a period of $10 \mathrm{~s}$ to $5 \mathrm{~min}$ by applying short pressure pulses $(50-80 \mathrm{psi}, 50 \mathrm{~ms}$ duration, $0.5-1.0 \mathrm{~Hz}$ ). In DLM/Area X recordings, single or multiunit Area X activity was recorded with a tungsten electrode placed $400-500 \mu \mathrm{m}$ posterior to the infusion pipette (i.e., in between the infusion pipette and DLM) (supplemental Fig. $1 \mathrm{~A}$, available at www.jneurosci.org as supplemental material) to monitor the effect of GABA on Area X neurons. In the first GABA infusion for each DLM neuron, GABA infusion was stopped as soon as spontaneous firing of either Area X or DLM neurons was noticeably changed (10-120 s). In the subsequent infusions, since it was unlikely that GABA could spread directly to the thalamic nucleus DLM from the infusion site in the anterior forebrain, we often continued to infuse GABA even when Area X activity was suppressed, until DLM spontaneous activity changed ( $10 \mathrm{~s}$ to $5 \mathrm{~min}$ ). In LMAN/Area $\mathrm{X}$ recordings, the Area X recording electrode was placed 400-500 $\mu \mathrm{m}$ dorsal to the infusion pipette (i.e., in between the infusion pipette and LMAN) (supplemental Fig. $1 B$, available at www.jneurosci.org as supplemental material). To avoid the drug directly spreading to LMAN, which is close to Area X, GABA infusion was stopped as soon as spontaneous firing of either Area X or LMAN neurons was noticeably changed (10-120 s). In 
both DLM/Area X and LMAN/Area X recordings, an effort was made to infuse GABA more than one time for each neuron, at 15-40 min intervals, to make sure that the effects were consistent and reliable.

The infusion site was labeled by $5 \%$ rhodamine dextran amine (3000 MW; Invitrogen) or 3\% pontamine sky blue (BDH Laboratory Supplies) dissolved in the GABA solution to confirm that the site was in Area X. In preliminary experiments, we estimated the spread of GABA solution using those dyes, but by monitoring Area $\mathrm{X}$ activity at a known distance from the GABA infusion site, we found that the dye spread was not necessarily matched with the area where neural activity was suppressed by GABA. Thus, we estimated the GABA-affected area only by directly monitoring neural activity of Area X neurons $400-500 \mu \mathrm{m}$ away from the infusion site.

Data analysis. We analyzed the effects of GABA infusion into Area X on LMAN and DLM neurons only when we observed a decrease in spontaneous activity of Area $\mathrm{X}$ neurons that we were recording simultaneously. We collected single-unit activity in LMAN and DLM during $15 \mathrm{~s}$ trials separated by $15 \mathrm{~s}$ intervals. Changes in spontaneous firing rates in response to GABA infusion were analyzed by comparing firing rates in the baseline trials, which were the 8-10 trials immediately before GABA infusion, and those in GABA-response trials, which were defined as follows. In cases when GABA was infused for a shorter period than four trials $(2 \mathrm{~min})$, the GABA-response trials were set to be four trials. When GABA was infused over a longer period than four trials, the response trials were defined to be as the same length as the infusion period. Because most neurons showed a change in their spontaneous activity at varying times after the GABA infusion onset, the response trials were offset by estimates of the delay determined as follows: if two consecutive trials both had firing rates that were more or less than two times the SD of firing rates in the baseline trials, response trials were defined starting at the beginning of the two consecutive trials; if there were no such trials, response trials were defined starting at the onset trial of GABA infusion (i.e., no delay). The significance of differences in firing rates between baseline trials and GABA-response trials was determined using MannWhitney $U$ tests $(p<0.05)$. When GABA was infused multiple times for individual neurons, we analyzed the activity before and during the GABA infusion causing the largest change.

For paired recordings from DLM projection neurons and putative pallidal axon terminals with a single electrode, we examined whether our recordings had similar spiking patterns to those reported by Person and Perkel (2007), using their analysis. A spike-triggered average (STA) was calculated between the DLM and pallidal spikes, by recovering pallidal spike times in a $250 \mathrm{~ms}$ window before each DLM spike and plotting them relative to the time of the DLM spike. The occurrences of pallidal spikes were histogrammed in $5 \mathrm{~ms}$ bins, and the spike probability in individual bins was calculated [although Person and Perkel (2007) referred to this as the "average firing rate"].

In the paired recordings, we also examined the relationship between the pattern of pallidal firing and the probability of DLM spiking, by analyzing pallidal firing ISIs, as follows. In each recording, we examined all ISIs in the pallidal firing, and for each ISI, we measured the mean ISI in an immediately preceding time window of $50 \mathrm{~ms}$ duration ("pre-ISI") (see Fig. 2D). We plotted the pre-ISIs versus the corresponding ISIs for each cell, identifying those ISIs that were followed by DLM spikes and those that were not (Fig. 2E); such plots compare the features of pallidal firing associated with the presence or absence of DLM spiking. We also generated a measure reflecting local firing rate changes by calculating the ratio of each individual ISI to its corresponding pre-ISI (ISI/pre-ISI $\times$ 100 ); a value $>100$ indicates that the firing rate just preceding an ISI was higher than the firing rate corresponding to that ISI (i.e., firing rate decrease). We then compared this measure, which we called "percentage change in pallidal ISIs," between ISIs that were followed by DLM spikes and those that were not, to examine the statistics of ISI changes (i.e., firing rate changes) in pallidal activity preceding DLM spiking.

Significance of the effects of Area X inactivation on DLM and LMAN firing rates was determined using the Mann-Whitney $U$ test. Significance of the recovery of LMAN firing rates from the effects of Area X inactivation was determined using the Friedman test and a post hoc test (Nemenyi test). Significance of the effects on coefficients of variation in LMAN activity was determined using the Wilcoxon signed-ranks test. The Man-
n-Whitney $U$ test was also used to determine the significance of the correlation of the ISI changes in pallidal firing with DLM spiking. In all cases, the significance level $(\alpha)$ was set at 0.05 .

\section{Results}

We investigated activity propagation in the songbird basal ganglia-thalamocortical circuit known as the AFP, focusing on two steps in the pathway, the pallidothalamic connection from Area X to DLM and the subsequent thalamocortical connection to LMAN. We first present the data for Area X-DLM signaling and then investigate how LMAN responds to the same manipulation used to study DLM.

\section{DLM neurons fire after decelerations in pallidal firing, consistent with the postinhibitory rebound model}

Pallidal-like neurons in the basal ganglia structure Area X send GABAergic inhibitory projections to the thalamic target neurons in DLM, each of which receives only one, or at most two, giant perisomatic terminals from pallidum (Fig. 1C) (Luo and Perkel, 1999a,b). This specialized arrangement of presynaptic and postsynaptic structures allowed Person and Perkel (2007) to simultaneously record a DLM soma and a (presumably synaptically coupled) pallidal axon terminal with a single extracellular electrode. By examining pallidal firing patterns in relation to DLM spikes in such paired recordings, they then found evidence suggesting that individual DLM spikes are preceded by a rapid deceleration in the rate of presynaptic pallidal firing. Because such pallidal firing patterns closely resemble the patterns of pallidal inhibitory inputs found to drive thalamic spikes via postinhibitory rebound in vitro (Person and Perkel, 2005), they raised the possibility that postinhibitory rebound is responsible for avian pallidothalamic signaling in vivo (postinhibitory rebound model) (Fig. $1 B_{1}$, intact circuit). In our further investigation of signaling mechanisms in this circuit, we first demonstrated that we could observe the same evidence consistent with the postinhibitory rebound model in our DLM soma/pallidal terminal recordings in vivo.

Just as in the recordings of Person and Perkel (2007), our paired recordings on a single electrode were typified by a largeramplitude unit with biphasic spike waveforms, from a putative DLM projection neuron, and a smaller-amplitude unit with relatively asymmetric spike waveforms, from a putative pallidal axon terminal (Fig. $2 A)$ ( $n=3$ paired recordings). In two of the three paired recordings, the larger units were also confirmed to project to LMAN using antidromic activation and collision tests (supplemental Fig. $2 \mathrm{~A}$, available at www.jneurosci.org as supplemental material). Analysis of spike times of pallidal firing with reference to each spontaneous DLM spike revealed a relationship between pallidal and DLM firing very similar to that reported by Person and Perkel (2007): the raster plot of pallidal firing shows a distinct gap just before DLM spiking (Fig. $2 B$, time 0 ), and thus the DLM STAs of pallidal firing have a sudden dip preceding DLM spiking (Fig. 2C); this decrease in STA often follows a slight increase in firing (Fig. 2C). These data are consistent with the postinhibitory rebound model, in which DLM spiking is driven by postinhibitory rebound, triggered by decelerations in highfrequency pallidal firing.

Since these STAs by definition represent the average probability of pallidal spiking before DLM spikes, they do not directly examine rate changes in pallidal firing before each individual DLM spike. Moreover, they do not reveal how pallidal firing that elicits DLM spiking differs from firing that does not. To address these issues, we investigated the local features of pallidal spike 
A

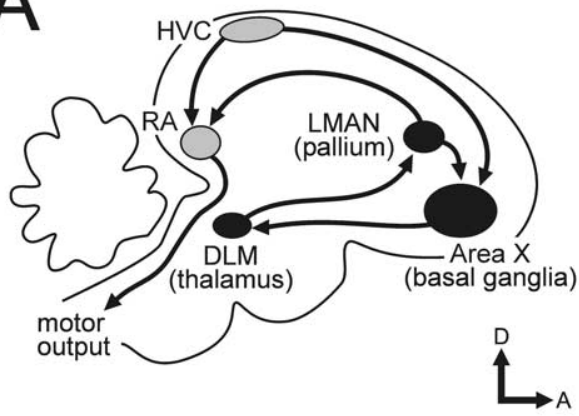

C

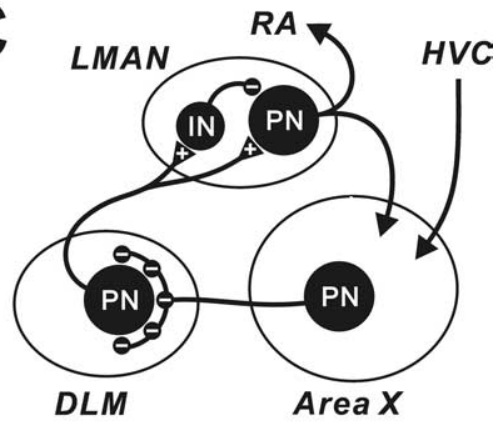

$\mathrm{B}_{1}$

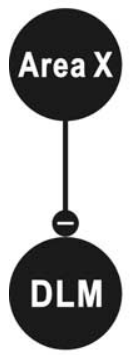

\section{Intact circuit}

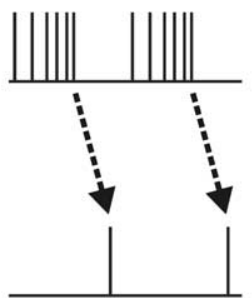

Postinhibitory rebound

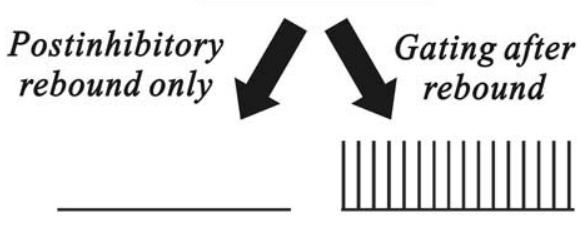

Postinhibitory

Figure 1. $A$, Schematic parasagittal view of the two major pathways in the zebra finch song system. The gray nuclei are in the vocal motor pathway, and the black nuclei are those of the AFP, an avian basal ganglia-thalamocortical circuit. $\boldsymbol{B}$, Hypothesized signaling mechanisms in the GABAergic inhibitory connection from pallidal-like Area $X$ neurons to thalamic relay neurons in DLM. In the intact circuit $\left(\boldsymbol{B}_{\boldsymbol{1}}\right)$, evidence suggests that DLM spikes are normally driven by postinhibitory rebound after rapid decelerations of Area X pallidal firing. If Area X neurons signal to DLM exclusively by postinhibitory rebound, inactivation of Area X activity will eliminate rebound-mediated spikes in DLM (because of loss of inhibitory input required to drive rebound), resulting in decreased DLM activity ( $\boldsymbol{B}_{\mathbf{2}}$, left). In contrast, if Area X neurons are capable of signaling to DLM by gating excitatory drive to DLM neurons, as well as by driving postinhibitory rebound, Area X inactivation will result in sustained firing in DLM (possibly after an initial rebound event) ( $\boldsymbol{B}_{\mathbf{2}}$, right). $\boldsymbol{C}$, Simplified representation of the major neural circuit in the AFP. Triangles with a plus sign indicate excitatory synapses, and small circles with a minus sign indicate inhibitory synapses; IN, interneuron; PN, projection neuron. Note that DLM projection neurons receive giant perisomatic terminals from Area X pallidal neurons.

trains that preferentially lead to DLM spiking, by analyzing the local changes in pallidal firing rates as a function of whether such changes resulted in DLM spiking or not. When we compared each individual pallidal ISI with the mean ISI in a $50 \mathrm{~ms}$ window preceding that ISI (Fig. $2 D$, pre-ISI), we found that most ISIs that were accompanied by DLM spiking (Fig. $2 E$, red dots in the example recording) were not only relatively long compared with the total ISI distribution but were also preceded by shorter pre-ISIs (i.e., by higher rates) than were ISIs not accompanied by DLM spiking (Fig. 2 E, black dots). To quantify this, we calculated the percentage change in ISIs between all the individual ISIs and their corresponding pre-ISIs (see Materials and Methods) in our paired recordings. This value was significantly higher before DLM spikes than was the same ratio before no spikes (Fig. $2 F$ ) ( $p<0.0001$ for all three recordings; Mann-Whitney $U$ test); moreover, the percentage changes before DLM spikes were mostly $>100 \%$ (i.e., increases in ISIs). These results indicate that rapid increases in pallidal ISI (i.e., rapid decelerations in pallidal firing) are more likely to elicit DLM spiking than are simply ISIs of a certain duration.

In addition, in the paired recordings with relatively low pallidal firing rates (for example, the one in Fig. $2 E$ ), the pre-ISIs followed by DLM spiking were predominantly shorter than the average ISI of overall firing (i.e., the mean rate): most red dots in
Figure $2 E$ lie below the dashed green line representing mean firing rate. This indicates that before DLM spiking, the pallidal firing rate first increases from the mean rate and then rapidly decreases. These DLM spiking properties, with their strong dependence on the local firing statistics of inhibitory pallidal input, are similar to those of DLM neurons known to be driven by postinhibitory rebound in vitro and thus provide additional evidence for the postinhibitory rebound model of pallidothalamic signaling.

We also found that DLM neurons generate mostly a single spike or a burst of a few spikes after each pallidal firing deceleration. This firing statistic was evident in the histogram of ISIs in DLM neurons (Fig. $2 G)(n=$ 14 neurons, three in paired recordings and 11 from separate recordings): the vast majority of DLM ISIs were longer than $50 \mathrm{~ms}$, the upper limit of the pallidal ISIs (Fig. 2E, abscissa), and there was a sharp peak of very short ISIs (mostly $<4 \mathrm{~ms}$ ) (Fig. $2 G$, red arrow), representing bursts (primarily of a small number of spikes). The characteristic of DLM neurons to fire at most a few spikes in a burst was also evident in the highly constant latency from the onset of spiking ISIs to DLM spiking (Fig. 2H) (SD of latency is $3.6 \pm 0.6 \mathrm{~ms}, n=3$ paired recordings); longer DLM bursts would give rise to much more jitter in the latency, because of the later spikes in the burst. The constant latency is seen also in the pallidal spike raster relative to DLM spikes shown in Figure $2 B$. The ISI distribution of our DLM spikes and the very tight pallidal-DLM spike latency are very similar to those of rebound spikes in DLM neurons in vitro (Person and Perkel, 2005), further supporting the idea that rebound could be responsible for pallidothalamic signaling in songbirds.

\section{DLM neurons increase their firing rate in response to Area $\mathrm{X}$ inactivation}

However, the characteristic presence of a deceleration in pallidal firing before DLM spiking, as described by Person and Perkel (2007) and above, does not by itself completely eliminate the possibility that pallidal neurons control DLM neurons by gating excitatory drive. As has been hypothesized for mammalian pallidothalamic signaling (MacLeod et al., 1980; Deniau and Chevalier, 1985; Inase et al., 1996), a DLM neuron with a strong separate source of excitatory drive (intrinsic or extrinsic) would also fire when released from inhibition by a pallidal firing pause, especially if the pause was relatively long (gating model). To address this possibility, we first proceeded to a direct test of whether silencing Area X pallidal projections to DLM can enable sustained activity in DLM neurons. We then also looked for evidence in birds in whom Area X was not being manipulated of spontaneous DLM firing patterns that would be consistent with gated excitation and not with postinhibitory rebound.

We silenced Area X pallidal activity acutely and reversibly by locally infusing GABA into Area $\mathrm{X}$ while monitoring extra- 
cellular activity of Area X neurons in anesthetized birds and simultaneously recording the activity of single DLM projection neurons. DLM projection neurons (11 neurons from 10 birds) were identified based on their very lowfrequency spontaneous firing rates $(0.0-$ 9.7 $\mathrm{Hz}$, mean $2.0 \mathrm{~Hz}$ ) and biphasic action potential spike waveforms (Fig. $3 B$, inset), following Person and Perkel (2007). We confirmed that six of those eleven units indeed projected to LMAN by antidromic activation and collision tests. In response to brief local GABA infusion into Area $X$ (the resulting Area $X$ inactivation is shown in Fig. $3 A$ ), most ( 8 of 11) DLM projection neurons exhibited a sustained increase in spontaneous firing rates (Fig. $3 B, C$ ). The increases in DLM firing rates were reversible and ended mostly within 5 min (i.e., 10 trials) after the end of GABA infusion $(p<0.0001$, Friedman test for the GABA effect; $p>$ 0.05 between baseline and post $5 \mathrm{~min}$, Nemenyi test) (Fig. 3D). The DLM response to GABA infusion was also reproducible: in six of the eight responding neurons, we infused GABA into Area X multiple times (2-3 times) at 15-30 $\mathrm{min}$ intervals and found a similar increase in firing rate after each infusion in five of the six neurons. When we infused GABA into Area X continuously even after Area $\mathrm{X}$ activity was completely shut down, DLM firing rates stayed high for a longer time (Fig. 3E, black line). These results are consistent with the gating model, in which DLM neurons fire in response to excitatory drive that is unmasked after loss of inhibitory inputs from Area X pallidal neurons.

In DLM, we also recorded 10 single units from eight birds that were putative axon terminals of Area X pallidal neurons, based on their distinctively higher spontaneous firing rates (mean, $81.9 \mathrm{~Hz}$; 37.7$139.7 \mathrm{~Hz}$ ) and relatively asymmetric (monophasic-like) spike waveforms (Fig. $4 A$, inset) (Person and Perkel, 2007). In a subset of these units $(n=3)$, we stimulated LMAN and never saw reliable antidromic spikes in DLM with fixed latencies after stimulation, consistent with the conclusion that those units are axon terminals of pallidal neurons. As expected, most of these units markedly decreased their spontaneous firing rates after GABA infusion into Area X ( 8 of 10 units), and no units increased their firing rates (Fig. $4 A, B$ ). In many units, spontaneous firing was completely suppressed and recovered to the baseline level within $5 \mathrm{~min}$ after the end of GABA infusions ( $p<0.001$, Friedman test for the GABA effect; $p>0.05$ between baseline and post $5 \mathrm{~min}$, Nemenyi test) (Fig. 4C), just like the recovery of DLM projection neurons from their tonic firing increase. The similar time scale of the GABA infusion ef-
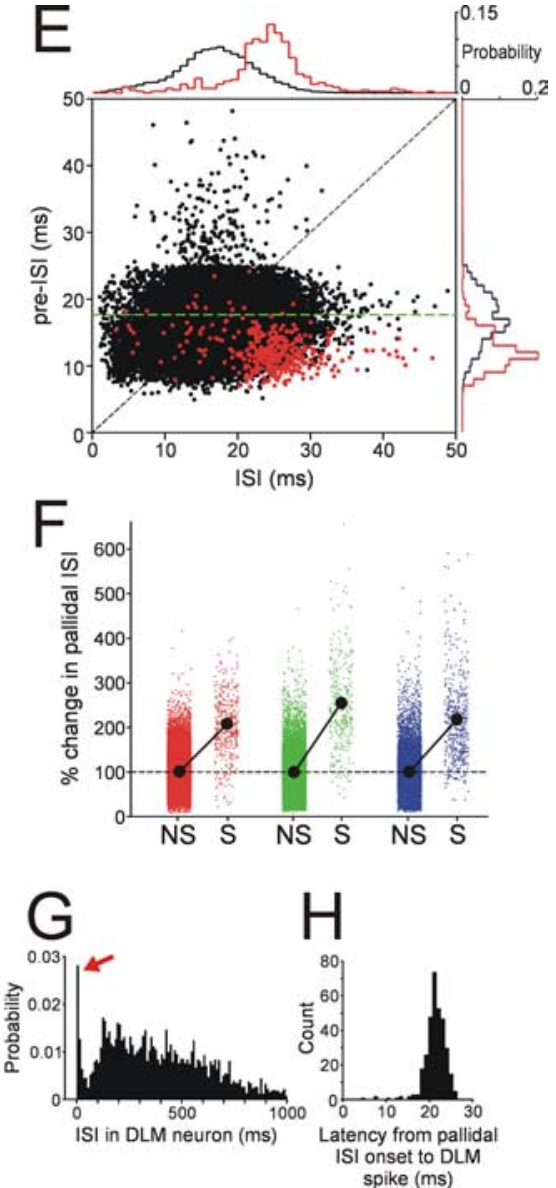

Figure 2. Firing decelerations in pallidal activity preceding DLM spikes provide evidence consistent with the postinhibitory rebound model. $\boldsymbol{A}$, Example of a simultaneous paired recording showing large DLM spikes occurring after rapid deceleration in pallidal firing (smaller spikes). Overlaid spike waveforms of the two units are shown in the inset; calibration: $0.5 \mathrm{~ms}$. $\boldsymbol{B}$, Raster of pallidal spike times with reference to each DLM spike, resembling the data from Person and Perkel (2007). The arrow points to a gap in the raster plot just before the DLM spikes at time 0. C, DLM spike-triggered average of pallidal firing for three paired recordings. In each recording, the probability of pallidal spike occurrence was calculated using $5 \mathrm{~ms}$ bins. The red line indicates the recording shown in $\boldsymbol{B}$. D, Schematic showing the analysis of rapid changes in pallidal firing rate. Pallidal spikes are represented by ticks on a flat line. Each ISI was compared with the mean ISI in a 50 ms time window preceding the ISI (pre-ISI) (see Materials and Methods). $\boldsymbol{E}$, ISIs in pallidal firing were plotted against pre-ISIs, as a function of whether the ISIs were accompanied by DLM spikes (red) or not (black), for the paired recording shown in $\boldsymbol{B}$. Probability distributions of the red and black dots projected along the axes are shown on the top and right (bin size, $1 \mathrm{~ms}$ ). The dashed diagonal line marks where a dot would lay if the ISI is equal to the mean ISI in the preceding period (i.e., no change in firing rate from the pre-ISI period to the ISI being considered). The dashed green line indicates the ISI corresponding to the overall mean firing rate of this pallidal unit (i.e., the ISI that is the inverse of the mean firing rate). $\boldsymbol{F}$, Percentage changes in pallidal ISIs (ISI/pre-ISI $\times 100)$ preceding DLM spikes $(S)$ and no spikes (NS). Different colors correspond to the different recordings in C. The filled circles connecting the two conditions indicate the mean of all ISI changes in each condition. The dashed line at the $100 \%$ ISI change indicates no change in ISIs between pre-ISI period and ISI. Note that in all the recordings, ISI changes followed by DLM spikes are predominantly larger than $100 \%$ (i.e., ISI increases and thus firing decelerations), and also in all cases significantly larger than the ISI changes followed by no DLM spikes ( $p<0.0001$ in all recordings, Mann-Whitney U test). G, Probability distribution of ISIs in DLM neurons ( $n=14$ neurons; 3 in paired recordings and 11 in separate recordings). The red arrow indicates a sharp peak representing multispike bursts. Bin size, $10 \mathrm{~ms}$. $\boldsymbol{H}$, Distribution of the latencies to a DLM spike from the preceding pallidal spike, calculated from the paired recording shown in $\boldsymbol{B}$. Bin size, $1 \mathrm{~ms}$.

fects between DLM neurons and pallidal axon terminals further supports the idea that loss of pallidal firing gates excitatory drive to DLM projection neurons and allows them to fire action potentials.

Increases in DLM firing rates are temporally matched with decreases in firing rates of simultaneously recorded pallidal axon terminals

To further investigate signaling in the pallidothalamic connection, we directly examined the relative timing of the increase in DLM firing rates and the decrease in pallidal firing rates after Area 

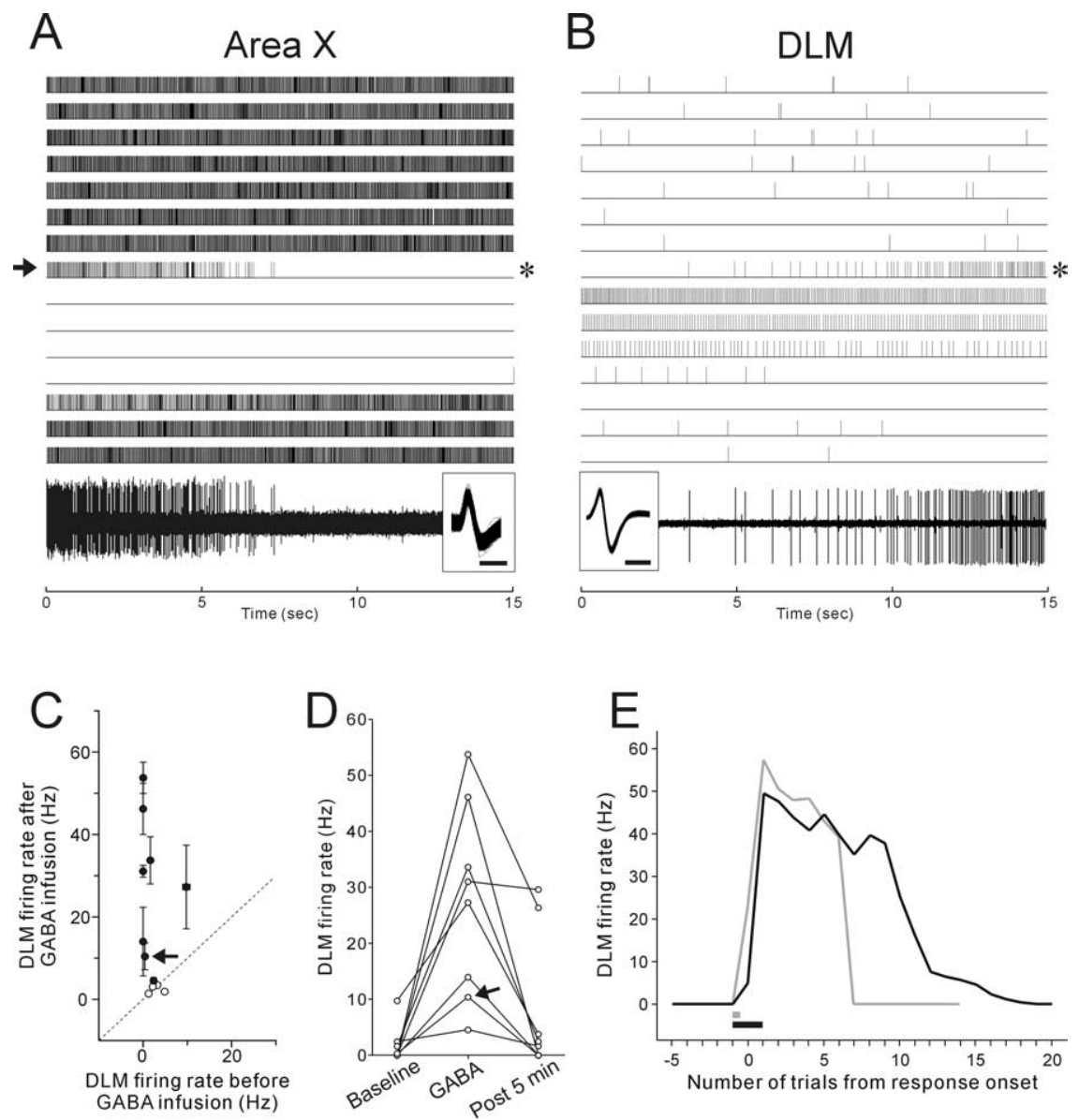

Figure 3. Effects of Area $X$ inactivation on Area $X$ and DLM activity. $A, B$, The raster (top) shows the single-unit activity of Area $X(\boldsymbol{A})$ and DLM $(\boldsymbol{B})$ neurons, recorded simultaneously with two different electrodes, responding to GABA infused into Area $X$ for $20 \mathrm{~s}$ (the arrow on the left side of the Area $X$ raster). Note that DLM activity increases as Area $X$ is inactivated by GABA infusion. The timing of GABA responses is not exactly matched because Area $X$ and DLM are topographically connected, with only $1-2$ Area $X$ neuron terminals per DLM neuron (Johnson et al., 1995; Luo et al., 2001), so that in simultaneous recordings of Area X and DLM the sites in each nucleus are related but not perfectly topographically matched. Raw traces of the Area X and DLM activity in the trials indicated by the asterisks on the right side of each raster are shown at the bottom with the overlaid spike waveforms in the inset; calibration: $0.5 \mathrm{~ms}$. C, Mean firing rates before GABA infusion were plotted against mean firing rates after GABA infusion for all DLM neurons tested. The GABA-response trials after GABA infusion were defined in individual neurons depending on GABA infusion time (see Materials and Methods). The diagonal line marks where cells would lay if mean firing rates before and after GABA infusion were equal. The filled circles indicate that the differences are statistically significant (Mann-Whitney $U$ test, $p<$ 0.05). The arrows in $\boldsymbol{C}$ and $\boldsymbol{D}$ indicate the neuron shown in $\boldsymbol{B}$. The majority of neurons showed a significant increase in firing rates after GABA infusion. $\boldsymbol{D}$, Recovery of LMAN firing rates after the GABA responses. For all DLM neurons that exhibited significantly increased activity after GABA infusion, firing rates before GABA infusion (baseline), in the GABA-response trials (GABA), and 5 min (i.e., 10 trials) after the end of GABA infusion (post $5 \mathrm{~min}$ ), are shown. $E$, Time courses of firing rate changes in a single DLM neuron after different GABA infusion times. Gray and black lines indicate firing rate changes after GABA infusions for 30 and $90 \mathrm{~s}$, respectively. The infusion periods are indicated as bars with corresponding colors. Note that DLM firing rates stayed high for longer if the duration of GABA infusion into Area $X$ was increased.

X inactivation, in simultaneous paired recordings of a DLM soma and a pallidal axon terminal with a single electrode (such as the example shown in Fig. 2).

Just as in the DLM projection neurons and pallidal axon terminals recorded separately, the two types of units in the paired recordings exhibited opposite effects of Area X inactivation on their spontaneous activity $(n=3$ paired recordings): the larger slow-firing units from DLM projection neurons increased their firing rates (Fig. $5 \mathrm{~A}$, red raster; the larger unit in Fig. $5 B$ ), whereas the smaller fast-firing units from pallidal axon terminals decreased their firing rates (Fig. 5A, blue raster; the smaller unit in Fig. 5B). The time courses of the increased and decreased effects were perfectly matched: as the pallidal firing rates started decreasing, the firing rate in the DLM projection neurons started increasing (Fig. 5A, right); the time courses of firing rate recovery were also matched between each of the two units. This result indicates that DLM projection neurons increase their firing rates in response to a decrease in the firing rate of the putative presynaptic terminals of Area X pallidal neurons, further supporting the gating model for pallidothalamic signaling.

Moreover, the discharge of DLM spikes in the paired recordings was strongly dependent on the pallidal firing rates on an even shorter time scale. In the transition period in which the DLM projection neuron started to fire tonically (Fig. $5 A, B$, trial \#2), the number of DLM spikes occurring between two consecutive pallidal spikes was strongly and highly significantly correlated with the interval between those two pallidal spikes: that is, as the pallidal neuron increased its interspike intervals (i.e., decreased its instantaneous firing rate), from $\sim 10 \mathrm{~ms}$, through $50 \mathrm{~ms}$, and up, the putative postsynaptic DLM neuron gradually and systematically fired more action potentials during the pallidal spike intervals (Fig. 5C) ( $r$ value for the fit to a line: 0.95 , with a slope of 1 DLM spike/27.4 ms pallidal ISI). The same relationship held true for each of the other pairs recorded $(r=0.78$, 1 spike/ $12.3 \mathrm{~ms}$, and $r=0.96,1$ spike/15.5 $\mathrm{ms})$. This provides further evidence that these DLM spikes were generated by excitatory drive that was increasingly revealed as the pallidal GABAergic input slowed, rather than by postinhibitory rebound.

Two of these three paired recordings were from cells that were also documented to show rapid decelerations in pallidal firing preceding DLM spiking (Fig. 2), before Area $\mathrm{X}$ inactivation. Thus, although such spike-triggered averages have been proposed to be a signature of DLM firing driven by postinhibitory rebound, the same DLM neurons can generate spikes that reflect excitation unmasked by pauses in pallidal firing.

\section{DLM neurons show evidence of gating-mediated signaling during spontaneous firing}

The results of our Area X inactivation experiment demonstrate that pallidal neurons can gate DLM activity, strongly inhibiting the nucleus when firing normally and then, when silenced, releasing underlying excitatory drive that induces tonic firing in DLM neurons. In the intact pallidothalamic circuit (i.e., with Area X active), however, most DLM spikes are preceded by decelerations in pallidal firing (Fig. 2) (Person and Perkel, 2007), and those spikes seem likely (although not yet conclusively) to be generated via postinhibitory rebound. Is there any evidence for gatingmediated signaling in the normally functioning circuit? We reasoned that trains of maintained, nonbursting spontaneous DLM 


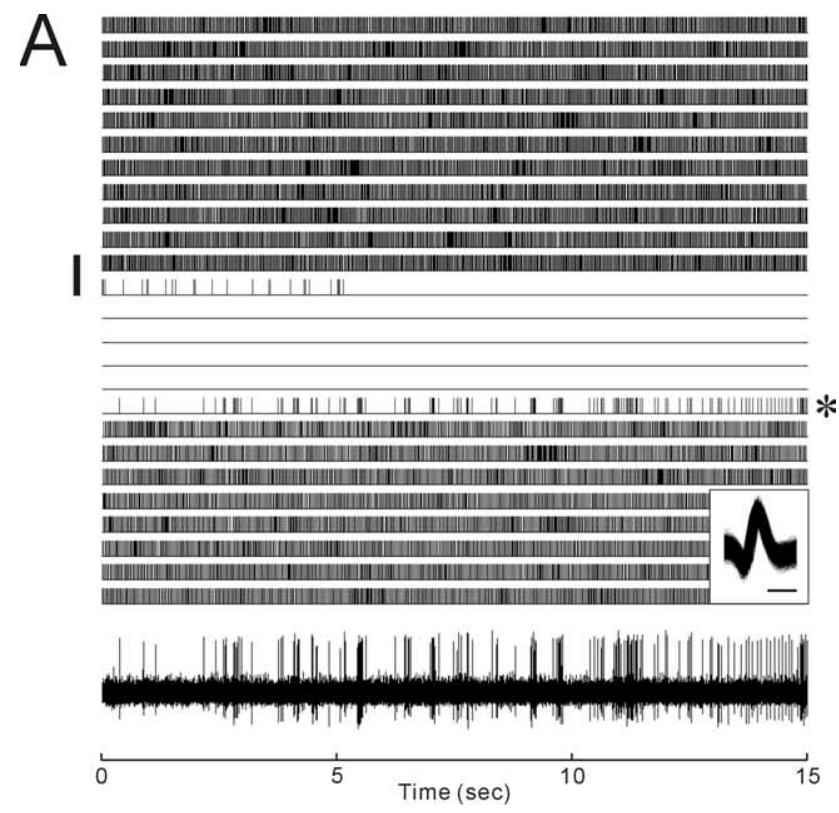

B

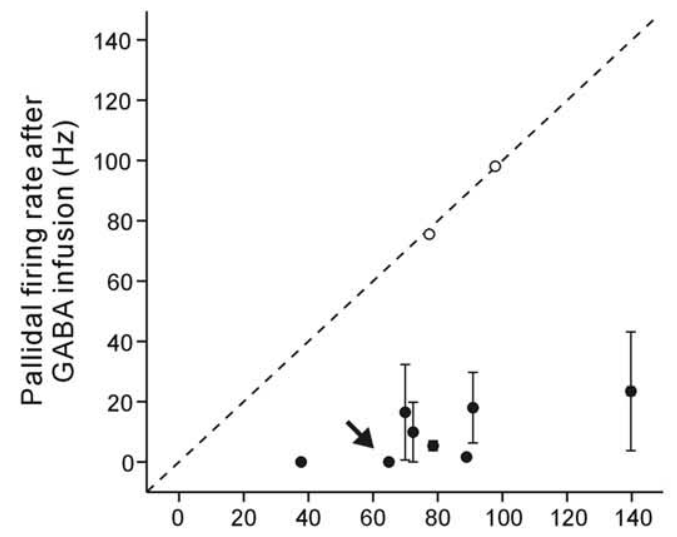

Pallidal firing rate before GABA infusion $(\mathrm{Hz})$
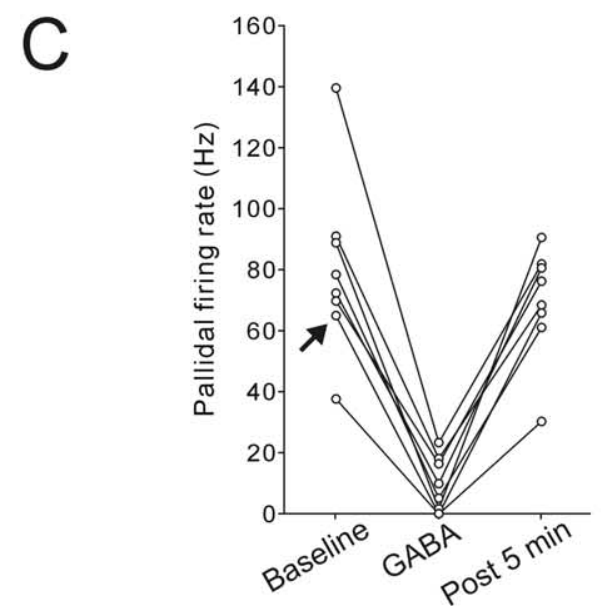

Figure 4. Decreased spontaneous activity in axon terminals of Area $X$ pallidal neurons in DLM after Area $X$ inactivation by GABA infusion. $\boldsymbol{A}$, The raster shows the responses of a single pallidal axon terminal to GABA infused into Area $X$ for $45 \mathrm{~s}$ (vertical bar). A raw trace of the pallidal axon activity from the trial indicated by the asterisk on the right side of the raster is shown at the bottom. Overlaid spike waveforms are shown in the inset; calibration: $0.2 \mathrm{~ms} . \boldsymbol{B}$, Mean firing rates before GABA infusion were plotted against mean firing rates after GABA infusion for pallidal axon activity. Conventions are as in Figure 3C. C, Recovery of firing rate changes in pallidal axon terminals that exhibited significant decreased activity after GABA infusion. Conventions are as in Figure $3 D$. firing would be consistent with firing gated by pallidal inhibition and not easily explained by postinhibitory rebound. We therefore searched for such firing patterns during the time before GABA infusion, both in DLM neurons recorded alone, and, when available, in DLM and pallidal neurons recorded simultaneously. Our criteria for firing likely to reflect gating were based on the following properties of DLM spiking. IPSP-driven rebound observed in vitro usually results in a short, high-frequency $(>250 \mathrm{~Hz}$; i.e., ISI $<4 \mathrm{~ms}$ ) burst consisting of mostly 1-2 DLM spikes (Person and Perkel 2005), and both our own and Person and Perkel's (2007) data suggest that the pattern of DLM spiking is similar in anesthetized birds in vivo. In addition, given the firing properties of rebound bursts in vitro, it has been estimated that individual rebound bursts at in vivo temperatures $\left(\sim 40^{\circ} \mathrm{C}\right)$ cannot be evoked at faster than $50 \mathrm{~Hz}$ : that is, each rebound burst must be followed by at least a $20 \mathrm{~ms}$ pause and coincident hyperpolarization to reset the rebound mechanism (Person and Perkel, 2005). Therefore, runs of five DLM spikes or more for which all ISIs are $<20 \mathrm{~ms}$, and for which at least one ISI is longer than $4 \mathrm{~ms}$ (so that the spikes do not represent a single burst event at $250 \mathrm{~Hz}$ or more), are unlikely to reflect postinhibitory rebound.

We found examples of such firing patterns in almost half our DLM projection neurons ( $n=6$ of 14 neurons; 38 episodes; the average firing rate within the sustained firing was $145.6 \pm 9.0 \mathrm{~Hz}$, the average duration of the sustained firings was $113.1 \pm 9.8 \mathrm{~ms}$ ) (Fig. $5 D_{1}$ ). Moreover, in two cases, these brief runs of sustained firing were from paired pallidothalamic recordings $(n=2)$, which showed that these episodes were indeed accompanied by long pauses in pallidal firing (Fig. $5 D_{2}$ ). This result provides evidence for intact DLM firing that is likely to be evoked by gating of excitatory drive in DLM neurons. Intriguingly, some examples show a brief burst followed at a short delay by more tonic firing (Fig. $5 D_{2}$, right), consistent with the idea that postinhibitory rebound is followed by unmasking of gated excitatory activity (see Discussion). Together, these findings raise the possibility that both gating and rebound contribute to the function of the normal circuit.

Although DLM neurons exhibited sustained firing consistent with gating of excitation, the occurrences of such firing in the spontaneous activity of our anesthetized birds were very rare. We observed only 0.13 episodes per minute on average across all neurons (range, $0-0.86$ episodes per minute for individual neurons), and thus the average fraction of spikes in the sustained firing epochs relative to all spikes recorded was also small (1.1\%). This suggests that much of pallidothalamic signaling in anesthetized birds, at least during spontaneous activity, is mediated by postinhibitory rebound, consistent with the STA analysis of pallidothalamic paired recordings shown in Figure 2.

Area $\mathrm{X}$ inactivation increases and regularizes the firing rate of LMAN neurons in adult birds

Like mammalian thalamic neurons, DLM neurons project to a pallial or cortical structure, LMAN, which in turn projects to the song premotor nucleus RA (Fig. 1A) (Okuhata and Saito, 1987; Bottjer et al., 1989). How does the gating-mediated increase in DLM activity influence the firing of LMAN neurons and their output to RA? DLM neurons send a glutamatergic excitatory projection to RA-projecting LMAN neurons (Fig. 1C), and thus tonic activation of DLM neurons may increase the activity of LMAN projection neurons (Fig. 6A, "excitation model"). However, LMAN projection neurons receive not only monosynaptic excitatory input from DLM but also a strong disynaptic inhibitory (GABAergic) input via LMAN interneurons (Fig. 1C) (Liv- 

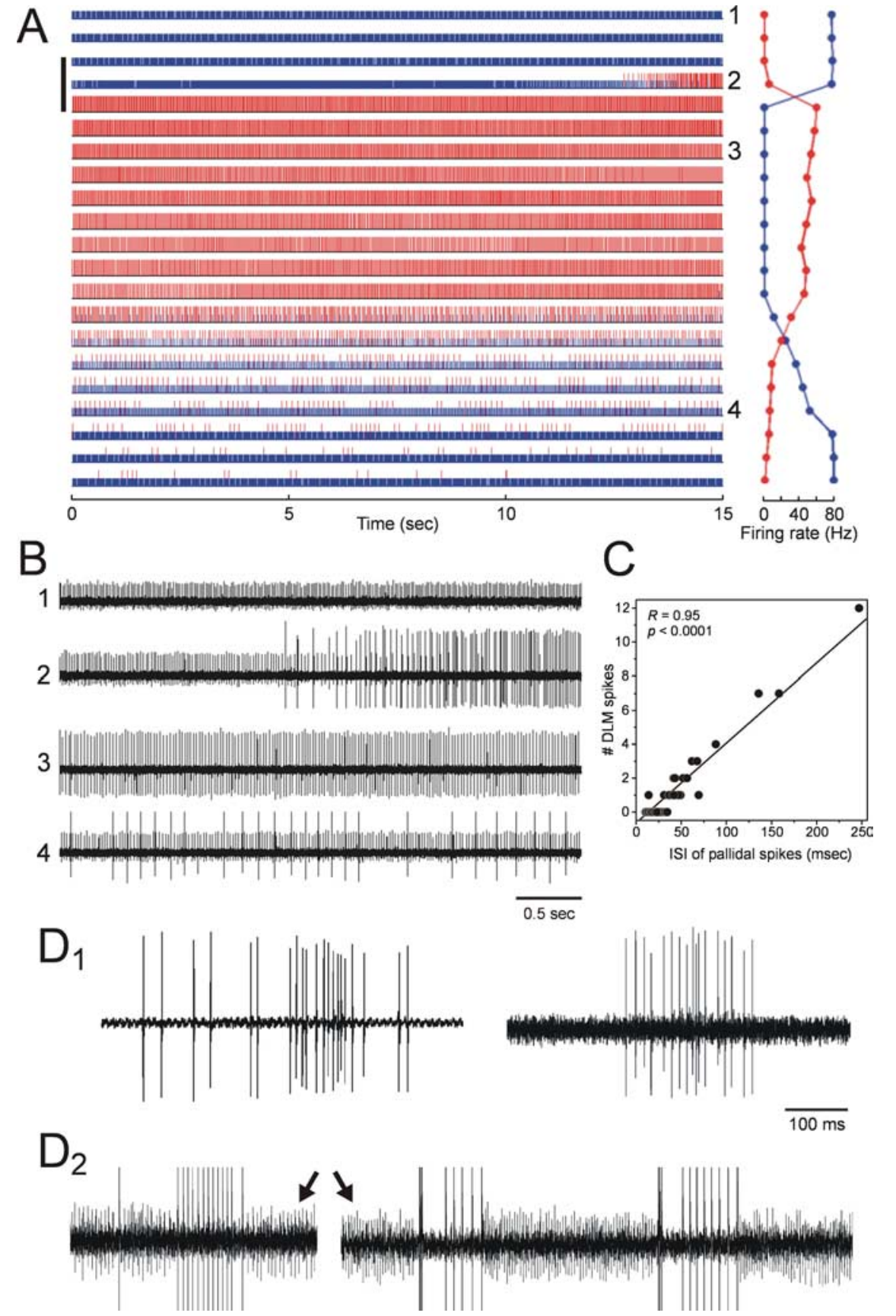

Figure 5. Simultaneous paired recordings of a DLM soma and an Area X pallidal terminal with a single electrode during Area X inactivation. $A$, The raster plots show the responses of DLM (red) and pallidal (blue) units, and GABA was infused into Area X over the period indicated by the vertical bar on the left (for $90 \mathrm{~s}$ ). Raw traces from the last $5 \mathrm{~s}$ of the numbered trials $(1-4)$ are shown in $\boldsymbol{B}$. The plots on the right side of the raster plots show mean firing rates of the DLM (red) and the pallidal (blue) units in each trial. Note that the time course of increased DLM activity after GABA infusion is completely matched with the decreased pallidal activity. This DLM unit had very low spontaneous activity before GABA infusion. C, Strong correlation between the duration of the ISI of pallidal firing and the number of DLM spikes occurring during the individual ISIs, calculated from the paired recording in the transition period during which the DLM unit started to fire tonically (trial $\# 2$ in $A$ and $B$ ). The line indicates the linear least-squares fit through the data and has an $r$ value of 0.95 and a slope of 1 DLM spike/27.4 ms pallidal ISI. D, Examples of spontaneous sustained firing of DLM spikes from two different single DLM recordings $\left(\boldsymbol{D}_{\boldsymbol{1}}\right)$ and one paired DLM soma-pallidal terminal recording $\left(\boldsymbol{D}_{\mathbf{2}}\right)$. In the paired recording, DLM spikes are truncated to enhance the visibility of the pallidal spikes (arrows). Note that the trains of high-frequency DLM firing were accompanied by pauses in pallidal firing.

ingston and Mooney, 1997; Bottjer et al., 1998). This inhibitory input evokes relatively large and long-lasting IPSPs in LMAN projection neurons in vitro when DLM afferents are strongly activated and thus could overcome excitatory inputs when DLM afferents are tonically activated at high frequency. If so, un- masked excitation of DLM neurons could actually decrease the activity of LMAN projection neurons (Fig. 6A, "inhibition model"). To directly examine these ideas, we recorded the activity of LMAN projection neurons while inactivating Area X.

Most LMAN neurons tested significantly increased their spontaneous firing rates after Area X inactivation ( 10 of 13 neurons; 7 of 8 birds) (Fig. $6 B-D$ ). Four of the ten LMAN neurons were identified as RA-projecting neurons using antidromic activation (supplemental Fig. $2 B$, available at www.jneurosciorg as supplemental material), and the other six neurons are also likely to be projection neurons because of their similar firing properties (see Materials and Methods). As in DLM neurons, the effects were usually rapid and ended within $5 \mathrm{~min}$ after the end of GABA infusion $(p<0.001$, Friedman test for the GABA effect; $p>0.05$ between baseline and post $5 \mathrm{~min}$, Nemenyi test) (Fig. $6 \mathrm{D}$ ). The LMAN response to GABA infusion was also reproducible: in eight of the ten responding neurons, we infused GABA into Area X multiple times (2-3 times) at 15-40 min intervals and found a similar increase in firing rate after each infusion in six of the eight neurons. Thus, together with the DLM recordings after Area X inactivation, this result demonstrates that the loss of Area X pallidal activity induces a tonic increase in DLM activity, which in turn increases LMAN output activity to the vocal motor pathway.

In addition, we found that the coefficients of variation $(\mathrm{CV})$ of LMAN ISIs within each trial were significantly smaller in the GABA-response trials than in the baseline trials $(n=9 ; p<0.05$, Wilcoxon signedrank test) (Fig. 6E). This suggests that LMAN neurons not only increase their firing rates but have more regular firing patterns after Area X inactivation. Although these CVs consistently decreased after Area X inactivation, in many neurons they were still close to, or more than 1.0, indicating some irregularity of firing (Fig. 6B). This may be attributable to our incomplete inactivation of Area X, but could also reflect the contribution of the inhibitory connection to the LMAN firing pattern, intermittently interrupting the regular excitatory input to LMAN projection neurons.

\section{Discussion}

In this study, we investigated how basal ganglia activity is transferred to pallium or songbird "cortex" through thalamus, in the specialized avian cortical-basal ganglia circuit known as the AFP. Our data show that thalamic relay neurons in DLM, which have been hypothesized to generate spikes by rebound from tonic inhibition by Area X pallidal neurons (Person and Perkel, 2005, 2007), exhibit sustained firing 
when Area X is pharmacologically inactivated. This demonstrates that songbird pallidal neurons can gate excitatory drive in thalamus, revealing this excitation when their firing rate drops (Fig. $1 B_{2}$, right panel). We also found evidence of spontaneous DLM firing patterns that are consistent with gating of excitation and not postinhibitory rebound, suggesting that intact avian pallidothalamic signaling might involve gating, perhaps in addition to postinhibitory rebound. Moreover, we showed that the activation of DLM neurons revealed by Area X silencing is forwarded to the target of DLM, the cortical nucleus LMAN. These results strengthen the parallels between avian and mammalian basal ganglia circuits and suggest that models of information processing in basal gangliathalamocortical circuits in general may be improved by considering the possibility that both gating and rebound are in play.

\section{Activity propagation in avian basal ganglia-thalamocortical circuits} Mammalian pallidum is thought to signal primarily via gating excitatory drive to thalamus, based on experimental results showing that pharmacological inactivation of pallidum induces tonic increases in the firing rate of target thalamic neurons (MacLeod et al., 1980; Deniau and Chevalier, 1985; Inase et al., 1996). By providing very similar evidence, we demonstrate that gating is a signaling mechanism in the avian pallidothalamic connection as well. Since the apparent gating that we observed occurred in the same circuit and even in the same DLM neurons that displayed evidence consistent with rebound-mediated signaling, i.e., DLM spiking after a rapid deceleration in pallidal neuron firing, our data raise the possibility that pallidal neurons signal to thalamus via both gating and rebound.

Although deceleration-triggered firing is consistent with postinhibitory rebound (Person and Perkel, 2005, 2007), it could also be generated by brief gating of tonic excitatory drive, allowing the appearance of spikes. However, our data along with that from other studies of the Area X-DLM connection provide additional support for a rebound model. For one, our analysis of pallidal ISI-triggered firing suggests that pallidal pauses preceded by a short period of high firing rates (which should hyperpolarize DLM neurons) are more likely to elicit DLM spikes than pauses of the same duration preceded by lower firing rates (Fig. 2E). This is what would be expected from rebound DLM firing, because the low-threshold $\mathrm{Ca}^{2+}$ channels important for this mechanism require hyperpolarization for their de-inactivation (Coulter et al., 1989; McCormick and Pape, 1990; Luo and Perkel, 1999b). Moreover, the activity during stimulusevoked DLM firing is better predicted by the rapid deceleration of test).
Excitation model

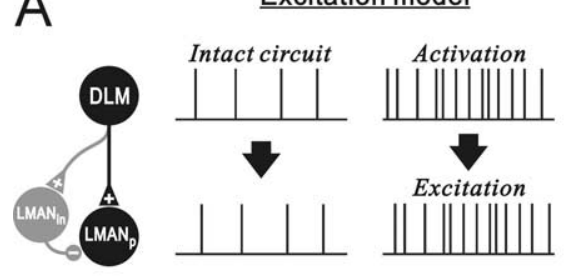

$\underline{\text { Inhibition model }}$

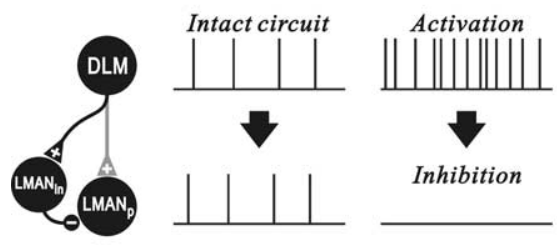

B

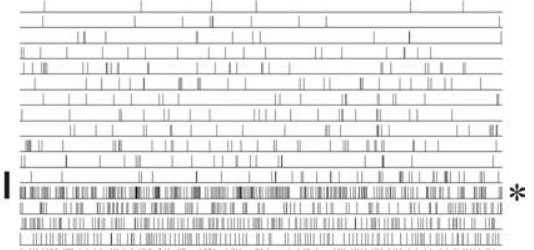

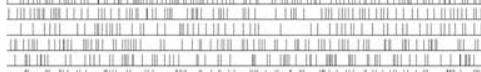

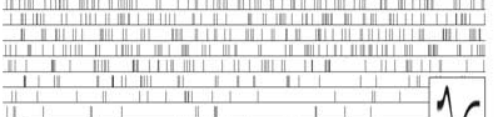

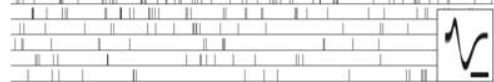

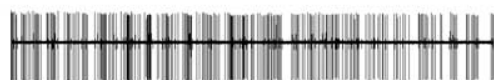

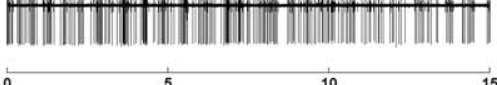
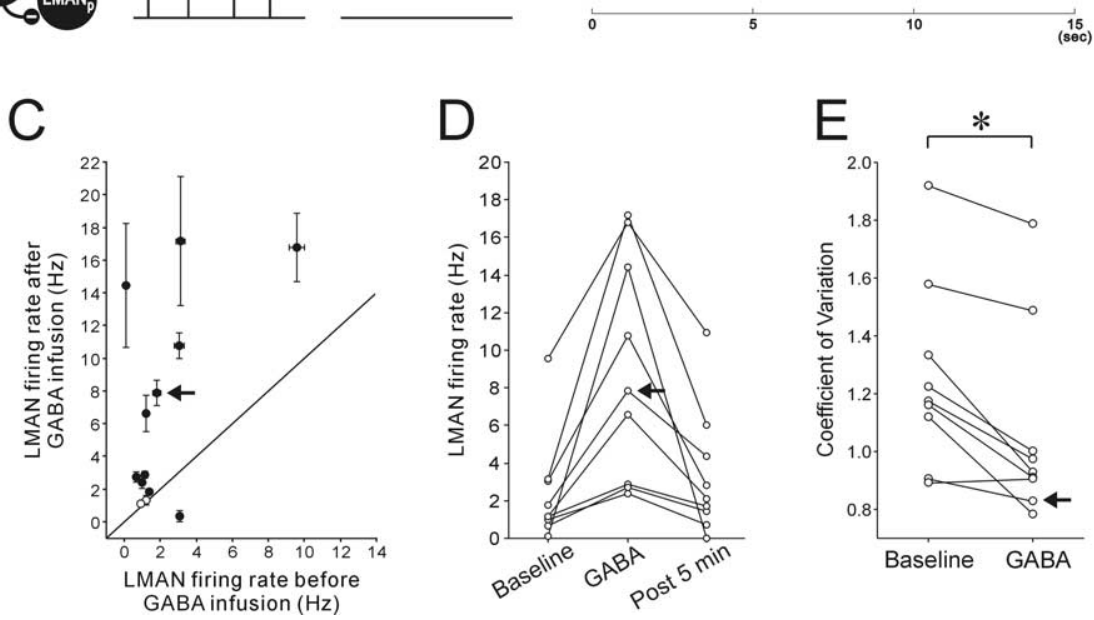

Figure 6. Increased activity in LMAN projection neurons after Area X inactivation by GABA infusion. $\boldsymbol{A}$, Two simple models for the effects of Area $X$ inactivation and resulting DLM activation on LMAN projection neurons. For each model, expected spike are shown with the neural pathway responsible for the LMAN activity. LMAN $_{\text {in }}$ LMAN interneuron; other conventions are as in Figure $1 B$. When the monosynaptic DLM excitatory projection to $L M A N_{p}$ is dominant relative to the polysynaptic inhibitory $\mathrm{LMAN}_{\mathrm{p}}$ will be inhibited by DLM. $\boldsymbol{B}$, The raster shows the responses of an LMAN projection neuron to GABA infused into Area $X$ for s (the vertical bar on the left). A raw trace of LMAN activity from the trials indicated by the asterisk on the right is shown at the 列 列 30 . $E$, The coefficient of variation of ISIs within trials was significantly decreased after GABA infusion. Two circles connected with a line illustrate each neuron. One neuron with a very low spontaneous firing rate $(<0.5 \mathrm{~Hz})$ was excluded from the analyses. Asterisks indicate significant differences between baseline and GABA conditions: * $p<0.05$ (Wilcoxon signed-rank

pallidal rates than by the length of pallidal pauses (Person and Perkel, 2007). Thus, pallidal neurons seem unlikely to signal to thalamus exclusively via gating of excitatory drive.

Given the potential for signaling via both rebound and gating, one possibility is that the circuit switches between these modes. One mechanism for such switching might involve the two distinct firing modes seen in DLM neurons in vitro, tonic and bursting, which, just as in other thalamic neurons (Jahnsen and Llinás, 1984; McCormick and Huguenard, 1992; Sherman, 2001; Bokor et al., 2005), depend on the cells' membrane potential. DLM neurons sitting at their resting potential can fire tonically throughout a depolarizing current pulse; when the same neurons are relatively hyperpolarized, voltage-dependent $\mathrm{Ca}^{2+}$ channels are de-inactivated and a depolarizing current pulse (or a rapid rise in membrane potential as inhibition is shut off) elicits a broad 
calcium spike crowned with a single or a few sodium spikes (Luo and Perkel, 1999b). When DLM neurons in vivo receive highfrequency pallidal inputs, they are likely to be hyperpolarized and thus in the bursting mode: in this mode, rapid decelerations in the high-frequency pallidal firing could cause a spike or burst of spikes by postinhibitory rebound. In contrast, when DLM neurons are more depolarized, either because of longer decreases in pallidal firing rate, Area X inactivation, or neuromodulatory influences, DLM neurons could switch to the tonic firing mode and produce tonic firing in response to excitatory drive, as discussed by Person and Perkel (2007). These burst and tonic modes of firing might explain the pattern of DLM spontaneous firing seen during some long pauses in pallidal firing (Fig. $5 D_{2}$, right): the first short burst may be a rebound-mediated burst caused by the abrupt termination of pallidal firing, and the subsequent regular firing may be gating-mediated tonic firing, resulting from a switch from the bursting to the tonic firing mode during the continued pause in pallidal firing. The possible contribution of both rebound and gating mechanisms, and their interaction, will ultimately require more direct investigation, such as intracellular recordings in vivo and pharmacologic manipulations of the relevant channels.

We found sustained DLM firing patterns that were inconsistent with postinhibitory rebound (Fig. 5D) in intact birds, but these were present in fewer than half of our DLM neurons, and then only at a very low rate. Thus, DLM spikes in anesthetized birds may be generated predominantly by postinhibitory rebound and not by a gating mechanism. This could reflect the firing mode of DLM neurons in anesthetized birds: DLM neurons may usually be in the burst firing mode and switch to the tonic firing mode more frequently only in awake birds. Indeed, a preliminary report on DLM projection neurons in awake juvenile birds described sustained firing with short ISIs ( $\sim 10 \mathrm{~ms}$ ) (Goldberg and Fee, 2007), which is not well explained by postinhibitory rebound.

Our results raise the question of where the DLM firing seen after pallidal inactivation originates. The tonic firing of mammalian thalamic neurons after the GABAergic afferent input from pallidum is decreased is attributed to the strong inputs to thalamus from cortex (Deniau and Chevalier, 1985). In songbirds, excitatory DLM neurons receive anatomical projections from the motor cortex homolog RA (Wild, 1993; Vates et al., 1997), which could, therefore, be the source of excitatory inputs that drive DLM spiking when pallidal inhibitory inputs are suppressed. Such an extrinsic excitatory mechanism for driving thalamus is supported by in vitro studies of DLM (Luo and Perkel, 1999b): robust glutamatergic EPSPs can be elicited in DLM by stimulating axonal fibers outside DLM. However, in anatomical studies, the axonal projection from RA to DLM is quite sparse, at least in the zebra finches studied here (Wild, 1993). Glutamatergic EPSPs in DLM in vitro could also be because of activation of recurrent collaterals, although the existence of recurrent collaterals has not been demonstrated (Luo and Perkel, 1999b). Moreover, RA spontaneous activity does not clearly drive thalamus, at least in anesthetized animals: RA and LMAN, which are interconnected via DLM, appear to be uncorrelated in their activity when their other interconnecting input, the vocal premotor nucleus HVC, is silenced (Kimpo et al., 2003) (a caveat is that RA input to DLM might have been masked in these experiments by tonic inhibition of DLM from Area X pallidal neurons). Given the current data, however, there is an intriguing possibility that when released from pallidal inhibition, DLM neurons fire spontaneously, or as a result of recurrent circuitry within the nucleus. Although DLM neurons show no resting spontaneous activity when they are intracellularly recorded in the slice, they sometimes fire spontaneously at $5-20 \mathrm{~Hz}$ during seal formation, before membrane rupture (Luo and Perkel, 1999b). In the long run, further experiments will be needed to determine the cause of tonic DLM firing during Area $\mathrm{X}$ inactivation, such as infusion of glutamate and/or GABA receptor blockers while recording DLM activity, and stimulation of putative sources of input.

We also examined the effects of pallidal silencing on LMAN, the cortical target of DLM. There has been little study of activity in cortex after pallidal silencing, despite the clinical importance of this question, except for imaging studies (Ceballos-Baumann et al., 1994; Grafton et al., 1995; Eidelberg et al., 1996; Samuel et al., 1997; Henselmans et al., 2000): these reveal that the hypoactivity of cortical motor and premotor areas seen in Parkinson's disease is normalized by pallidotomy. We provide here a neurophysiological correlate of these findings by showing that in response to Area $\mathrm{X}$ inactivation, projection neurons in the thalamic target LMAN increase and regularize their firing activity. Despite the strength of the feed-forward inhibitory projection to LMAN from DLM demonstrated in vitro (Livingston and Mooney, 1997; Bottjer et al., 1998), the increase in DLM firing after Area X inactivation, which can be up to $50 \mathrm{~Hz}$, appears to be transferred to LMAN primarily through the excitatory connection.

\section{Implications for the functions of basal ganglia circuits in songbirds and mammals}

The effects of Area X inactivation seen here also shed light on behavioral experiments on song learning and plasticity. In juvenile birds in the sensorimotor phase of song learning, lesioning or transiently inactivating the AFP output nucleus LMAN disrupts normal song development and induces premature song stereotypy. In contrast, lesioning the AFP input nucleus Area X prevents normal song crystallization and often increases the overall variability of song structure (Bottjer et al., 1984; Sohrabji et al., 1990; Scharff and Nottebohm, 1991; Olveczky et al., 2005). This striking difference in the effects of AFP interruption on song at different levels of the circuit can be accounted for by the difference in AFP output activity demonstrated by our results. Lesions of Area X, just like inactivation, may increase the activity of LMAN projection neurons (transiently or chronically); this could maintain or increase variability in song motor patterns and subsequent vocal output by continuously activating neurons in the song premotor nucleus RA. Such activation of RA neurons would presumably be caused by the increased excitatory synaptic inputs from LMAN (Olveczky et al., 2005), although other signals such as neurotrophic factors have also been suggested to be involved (Johnson et al., 1997; Kittelberger and Mooney, 1999, 2005). In contrast to Area X lesions, LMAN lesions eliminate all possible signals that RA receives from the AFP, including synaptic input and neurotrophic factors and thus may cause song to become more stereotyped.

Although the striking effects of Area X lesions on juvenile song can be accounted for, at least in part, by our data, the same lesions in adult birds have much more subtle effects on song. No dramatic changes are found after Area $\mathrm{X}$ lesions in overall acoustic structure or variability of adult song (Sohrabji et al., 1990; Scharff and Nottebohm, 1991), except for an increase in the number of repeated syllables in particular songs (Kubikova et al., 2007). The mild effect of Area X lesions on adult song, despite the tonic increase in LMAN activity observed here in the absence of Area X, could be attributable to decreased susceptibility of the song motor pathway to AFP output activity in adult birds (Brainard and 
Doupe, 2001; Olveczky et al., 2005; Kao and Brainard, 2006). Although adult LMAN neurons are able to direct real-time changes in song structure (Kao et al., 2005), such change occurs when these neurons are stimulated with brief pulses of activity systematically related to song. In contrast, the relatively regular and maintained LMAN firing that we observed after Area X inactivation may be insufficient to drive dramatic changes in adult $\mathrm{RA}$ and in song. It is also possible that Area X lesions have effects on aspects of song structure, such as song stereotypy, that have not been detected in previous studies. Further work is needed to establish how Area X lesions modify AFP output activity and influence song motor output in both juvenile and adult birds.

Our data also have implications for the functions of mammalian basal ganglia-thalamocortical circuits. If dual modes of signaling from pallidum to thalamus exist in mammalian basal ganglia as well, this could explain some of the complexities and apparent paradoxes in the properties of both normal and abnormal firing in such circuits. For instance, pallidotomy and highfrequency stimulation of pallidum both relieve parkinsonian symptoms, despite being apparently opposite manipulations (Graham et al., 1990; Lozano et al., 1995; Kumar et al., 1998; Benabid, 2003; Israel and Bergman, 2008). Pallidal ablation might increase circuit firing, regularizing it and eliminating pathological bursting, acting via gating like that observed here. Changes in pallidal activity caused by high-frequency stimulation (Bar-Gad et al., 2004), however, might equivalently normalize activity and eliminate pathological firing by driving temporally patterned increases in circuit firing via postinhibitory rebound. Similarly, the prominent oscillations accompanied by spike bursts and pauses found in movement disorders (Filion and Tremblay, 1991; Bergman et al., 1994; Wichmann et al., 1999; Soares et al., 2004) could have very different effects on motor output depending on whether the circuit was in a mode favoring rebound or gating. Since mammalian pallidal neurons and other GABAergic afferents to thalamus form large terminal plexi on thalamus neurons (Parent et al., 2001; Barthó et al., 2002; Bodor et al., 2008), similar to the calyx-like synapses in DLM, our results suggest that parallel examination of pallidothalamic signaling in mammals will provide general insights into the functions of basal ganglia-thalamocortical circuits and the pathophysiology of motor disorders.

\section{References}

Bar-Gad I, Elias S, Vaadia E, Bergman H (2004) Complex locking rather than complete cessation of neuronal activity in the globus pallidus of a 1-methyl-4-phenyl-1,2,3,6-tetrahydropyridine-treated primate in response to pallidal microstimulation. J Neurosci 24:7410-7419.

Barthó P, Freund TF, Acsády L (2002) Selective GABAergic innervation of thalamic nuclei from zona incerta. Eur J Neurosci 16:999-1014.

Benabid AL (2003) Deep brain stimulation for Parkinson's disease. Curr Opin Neurobiol 13:696-706.

Bergman H, Wichmann T, Karmon B, DeLong MR (1994) The primate subthalamic nucleus. II. Neuronal activity in the MPTP model of parkinsonism. J Neurophysiol 72:507-520.

Bodor AL, Giber K, Rovó Z, Ulbert I, Acsády L (2008) Structural correlates of efficient GABAergic transmission in the basal ganglia-thalamus pathway. J Neurosci 28:3090-3102.

Boettiger CA, Doupe AJ (1998) Intrinsic and thalamic excitatory inputs onto songbird LMAN neurons differ in their pharmacological and temporal properties. J Neurophysiol 79:2615-2628.

Bokor H, Frère SG, Eyre MD, Slézia A, Ulbert I, Lüthi A, Acsády L (2005) Selective GABAergic control of higher-order thalamic relays. Neuron 45:929-940.

Bottjer SW, Johnson F (1997) Circuits, hormones, and learning: vocal behavior in songbirds. J Neurobiol 33:602-618.

Bottjer SW, Miesner EA, Arnold AP (1984) Forebrain lesions disrupt devel- opment but not maintenance of song in passerine birds. Science 224:901-903.

Bottjer SW, Halsema KA, Brown SA, Miesner EA (1989) Axonal connections of a forebrain nucleus involved with vocal learning in zebra finches. J Comp Neurol 279:312-326.

Bottjer SW, Brady JD, Walsh JP (1998) Intrinsic and synaptic properties of neurons in the vocal-control nucleus IMAN from in vitro slice preparations of juvenile and adult zebra finches. J Neurobiol 37:642-658.

Brainard MS, Doupe AJ (2001) Postlearning consolidation of birdsong: stabilizing effects of age and anterior forebrain lesions. J Neurosci 21:2501-2517.

Ceballos-Baumann AO, Obeso JA, Vitek JL, Delong MR, Bakay R, Linazasoro G, Brooks DJ (1994) Restoration of thalamocortical activity after posteroventral pallidotomy in Parkinson's disease. Lancet 344:814.

Coulter DA, Huguenard JR, Prince DA (1989) Calcium currents in rat thalamocortical relay neurones: kinetic properties of the transient, lowthreshold current. J Physiol 414:587-604.

Deniau JM, Chevalier G (1985) Disinhibition as a basic process in the expression of striatal functions. II. The striato-nigral influence on thalamocortical cells of the ventromedial thalamic nucleus. Brain Res 334:227-233.

Eidelberg D, Moeller JR, Ishikawa T, Dhawan V, Spetsieris P, Silbersweig D, Stern E, Woods RP, Fazzini E, Dogali M, Beric A (1996) Regional metabolic correlates of surgical outcome following unilateral pallidotomy for Parkinson's disease. Ann Neurol 39:450-459.

Filion M, Tremblay L (1991) Abnormal spontaneous activity of globus pallidus neurons in monkeys with MPTP-induced parkinsonism. Brain Res 547:142-151.

Goldberg JH, Fee MS (2007) Contribution of the basal ganglia and related thalamus to song production in juvenile zebra finches. Soc Neurosci Abstr 33:430.25.

Grafton ST, Waters C, Sutton J, Lew MF, Couldwell W (1995) Pallidotomy increases activity of motor association cortex in Parkinson's disease: a positron emission tomographic study. Ann Neurol 37:776-783.

Graham WC, Robertson RG, Sambrook MA, Crossman AR (1990) Injection of excitatory amino acid antagonists into the medial pallidal segment of a 1-methyl-4-phenyl-1,2,3,6-tetrahydropyridine (MPTP) treated primate reverses motor symptoms of parkinsonism. Life Sci 47:PL91-PL97.

Hahnloser RH, Fee MS (2007) Sleep-related spike bursts in HVC are driven by the nucleus interface of the nidopallium. J Neurophysiol 97:423-435.

Hahnloser RH, Kozhevnikov AA, Fee MS (2002) An ultra-sparse code underlies the generation of neural sequences in a songbird. Nature 419:65-70.

Henselmans JM, de Jong BM, Pruim J, Staal MJ, Rutgers AW, Haaxma R (2000) Acute effects of thalamotomy and pallidotomy on regional cerebral metabolism, evaluated by PET. Clin Neurol Neurosurg 102:84-90.

Inase M, Buford JA, Anderson ME (1996) Changes in the control of arm position, movement, and thalamic discharge during local inactivation in the globus pallidus of the monkey. J Neurophysiol 75:1087-1104.

Israel Z, Bergman H (2008) Pathophysiology of the basal ganglia and movement disorders: from animal models to human clinical applications. Neurosci Biobehav Rev 32:367-377.

Jahnsen H, Llinás R (1984) Voltage-dependent burst-to-tonic switching of thalamic cell activity: an in vitro study. Arch Ital Biol 122:73-82.

Johnson F, Sablan MM, Bottjer SW (1995) Topographic organization of a forebrain pathway involved with vocal learning in zebra finches. J Comp Neurol 358:260-278.

Johnson F, Hohmann SE, DiStefano PS, Bottjer SW (1997) Neurotrophins suppress apoptosis induced by deafferentation of an avian motor-cortical region. J Neurosci 17:2101-2111.

Kao MH, Brainard MS (2006) Lesions of an avian basal ganglia circuit prevent context-dependent changes to song variability. J Neurophysiol 96:1441-1455.

Kao MH, Doupe AJ, Brainard MS (2005) Contributions of an avian basal ganglia-forebrain circuit to real-time modulation of song. Nature 433:638-643.

Kimpo RR, Theunissen FE, Doupe AJ (2003) Propagation of correlated activity through multiple stages of a neural circuit. J Neurosci 23:5750-5761.

Kittelberger JM, Mooney R (1999) Lesions of an avian forebrain nucleus that disrupt song development alter synaptic connectivity and transmission in the vocal premotor pathway. J Neurosci 19:9385-9398. 
Kittelberger JM, Mooney R (2005) Acute injections of brain-derived neurotrophic factor in a vocal premotor nucleus reversibly disrupt adult birdsong stability and trigger syllable deletion. J Neurobiol 62:406-424.

Kojima S, Doupe AJ (2007) Song selectivity in a pallial-basal ganglia song circuit of zebra finches raised without tutor song exposure. J Neurophysiol 98:2099-2109.

Kubikova L, Turner EA, Scharff C, Jarvis ED (2007) A predisposition to stutter and its recovery following basal ganglia damage. Soc Neurosci Abstr 33:430.6.

Kumar R, Lozano AM, Montgomery E, Lang AE (1998) Pallidotomy and deep brain stimulation of the pallidum and subthalamic nucleus in advanced Parkinson's disease. Mov Disord 13 [Suppl 1]:73-82.

Lewicki MS (1994) Bayesian modelling and classification of neural signals. Neural Comput 6:1005-1030.

Livingston FS, Mooney R (1997) Development of intrinsic and synaptic properties in a forebrain nucleus essential to avian song learning. J Neurosci 17:8997-9009.

Lozano AM, Lang AE, Galvez-Jimenez N, Miyasaki J, Duff J, Hutchinson WD, Dostrovsky JO (1995) Effect of GPi pallidotomy on motor function in Parkinson's disease. Lancet 346:1383-1387.

Luo M, Perkel DJ (1999a) Long-range GABAergic projection in a circuit essential for vocal learning. J Comp Neurol 403:68-84.

Luo M, Perkel DJ (1999b) A GABAergic, strongly inhibitory projection to a thalamic nucleus in the zebra finch song system. J Neurosci 19:6700-6711.

Luo M, Ding L, Perkel DJ (2001) An avian basal ganglia pathway essential for vocal learning forms a closed topographic loop. J Neurosci 21:6836-6845.

MacLeod NK, James TA, Kilpatrick IC, Starr MS (1980) Evidence for a GABAergic nigrothalamic pathway in the rat. II. Electrophysiological studies. Exp Brain Res 40:55-61.

McCormick DA, Huguenard JR (1992) A model of the electrophysiological properties of thalamocortical relay neurons. J Neurophysiol 68:1384-1400.

McCormick DA, Pape HC (1990) Properties of a hyperpolarizationactivated cation current and its role in rhythmic oscillation in thalamic relay neurones. J Physiol 431:291-318.

Mooney R, Rosen MJ, Sturdy CB (2002) A bird's eye view: top down intracellular analyses of auditory selectivity for learned vocalizations. J Comp Physiol A Neuroethol Sens Neural Behav Physiol 188:879-895.

Okuhata S, Saito N (1987) Synaptic connections of thalamo-cerebral vocal control nuclei of the canary. Brain Res Bull 18:35-44.

Olveczky BP, Andalman AS, Fee MS (2005) Vocal experimentation in the juvenile songbird requires a basal ganglia circuit. PLoS Biol 3:e153.

Parent M, Lévesque M, Parent A (2001) Two types of projection neurons in the internal pallidum of primates: single-axon tracing and threedimensional reconstruction. J Comp Neurol 439:162-175.

Paz JT, Chavez M, Saillet S, Deniau JM, Charpier S (2007) Activity of ventral medial thalamic neurons during absence seizures and modulation of cortical paroxysms by the nigrothalamic pathway. J Neurosci 27:929-941.
Person AL, Perkel DJ (2005) Unitary IPSPs drive precise thalamic spiking in a circuit required for learning. Neuron 46:129-140.

Person AL, Perkel DJ (2007) Pallidal neuron activity increases during sensory relay through thalamus in a songbird circuit essential for learning. J Neurosci 27:8687-8698.

Person AL, Gale SD, Farries MA, Perkel DJ (2008) Organization of the songbird basal ganglia, including area X. J Comp Neurol 508:840-866.

Reiner A, Perkel DJ, Mello CV, Jarvis ED (2004a) Songbirds and the revised avian brain nomenclature. Ann N Y Acad Sci 1016:77-108.

Reiner A, Perkel DJ, Bruce LL, Butler AB, Csillag A, Kuenzel W, Medina L, Paxinos G, Shimizu T, Striedter G, Wild M, Ball GF, Durand S, Güntürkün O, Lee DW, Mello CV, Powers A, White SA, Hough G, Kubikova L, et al. (2004b) Revised nomenclature for avian telencephalon and some related brainstem nuclei. J Comp Neurol 473:377-414.

Rosen MJ, Mooney R (2000) Intrinsic and extrinsic contributions to auditory selectivity in a song nucleus critical for vocal plasticity. J Neurosci 20:5437-5448.

Samuel M, Ceballos-Baumann AO, Turjanski N, Boecker H, Gorospe A, Linazasoro G, Holmes AP, DeLong MR, Vitek JL, Thomas DG, Quinn NP, Obeso JA, Brooks DJ (1997) Pallidotomy in Parkinson's disease increases supplementary motor area and prefrontal activation during performance of volitional movements an $\mathrm{H} 2(15) \mathrm{O}$ PET study. Brain 120:1301-1313.

Scharff C, Nottebohm F (1991) A comparative study of the behavioral deficits following lesions of various parts of the zebra finch song system: implications for vocal learning. J Neurosci 11:2896-2913.

Sherman SM (2001) Tonic and burst firing: dual modes of thalamocortical relay. Trends Neurosci 24:122-126.

Soares J, Kliem MA, Betarbet R, Greenamyre JT, Yamamoto B, Wichmann T (2004) Role of external pallidal segment in primate parkinsonism: comparison of the effects of 1-methyl-4-phenyl-1,2,3,6-tetrahydropyridineinduced parkinsonism and lesions of the external pallidal segment. J Neurosci 24:6417-6426.

Sohrabji F, Nordeen EJ, Nordeen KW (1990) Selective impairment of song learning following lesions of a forebrain nucleus in the juvenile zebra finch. Behav Neural Biol 53:51-63.

Solis MM, Doupe AJ (1999) Contributions of tutor and bird's own song experience to neural selectivity in the songbird anterior forebrain. J Neurosci 19:4559-4584.

Vates GE, Vicario DS, Nottebohm F (1997) Reafferent thalamo-"cortical” loops in the song system of oscine songbirds. J Comp Neurol 380:275-290.

Wichmann T, Bergman H, Starr PA, Subramanian T, Watts RL, DeLong MR (1999) Comparison of MPTP-induced changes in spontaneous neuronal discharge in the internal pallidal segment and in the substantia nigra pars reticulata in primates. Exp Brain Res 125:397-409.

Wild JM (1993) Descending projections of the songbird nucleus robustus archistriatalis. J Comp Neurol 338:225-241. 